\section{Efeito da acupuntura nas respostas de estresse em equinos atletas submetidos a reprise de adestramento}

\author{
Effect of acupuncture on stress responses in equine athletes \\ submitted to dressage
}

\author{
Julia Dias Villas-Boas ${ }^{1}$, Norma Aparecida dos Santos Almeida², Fernando Queiroz de Almeida ${ }^{3}$ \& \\ Magda Alves de Medeiros ${ }^{3,4 *}$ \\ 'Medica veterinária, Dr. Programa Multicêntrico de Pós-graduação em Ciências Fisiológicas, Universidade Federal Rural do Rio \\ de Janeiro - UFRRJ, Seropédica, RJ, Brasil \\ ²Bióloga, Dr. Departamento de Ciências Fisiológicas - DCF, Instituto de Ciências Biológicas e da Saúde - ICBS, Universidade \\ Federal Rural do Rio de Janeiro - UFRRJ, Seropédica, RJ, Brasil \\ ${ }^{3}$ Médicos veterinários, PhD. Departamento de Medicina e Cirurgia Veterinária, Instituto de Veterinária, Universidade Federal \\ Rural do Rio de Janeiro - UFRRJ, Seropédica, RJ, Brasil \\ ${ }^{4}$ Médica veterinária, Dr. Departamento de Ciências Fisiológicas - DCF, Instituto de Ciências Biológicas e da Saúde - ICBS, \\ Universidade Federal Rural do Rio de Janeiro - UFRRJ, Seropédica, RJ, Brasil
}

\section{Resumo}

O cavalo tem uma predisposição natural para o esporte, no entanto, o seu uso em competições pode resultar em problemas relacionados ao estresse que prejudicam seu desempenho esportivo e principalmente a sua saúde. Neste sentido éfundamental desenvolver estratégias que possam prevenir ou minimizar os efeitos deletérios do estresse. Neste sentido a acupuntura é uma técnica milenar da Medicina Tradicional Chinesa que tem sido utilizada no tratamento e prevenções de doenças relacionadas ao estresse. Desta forma, avaliamos se acupuntura pode alterar as respostas de estresse em cavalos atletas submetidos a uma prova de reprise de adestramento. Seis equinos da raça Brasileiro de Hipismo, com idade entre 6 e 8 anos, experientes em provas de adestramento, provenientes da Escola de Equitação do Exército, Rio de Janeiro, Brasil foram utilizados. O efeito da acupuntura nos pontos VG1, C7, VG2O e B52 imediatamente antes da reprise de adestramento foi avaliada através da análise do desempenho na prova, do cortisol sérico e da variabilidade da frequência cardíaca (VFC). Para análise da VFC foi utilizado frequencímetro cardíaco Polar Equine $^{\mathrm{TM}}$ e as amostras de sangue foram coletadas antes e 30 minutos após o fim da reprise para análise do cortisol. O desempenho dos animais foi avaliado por juízes oficiais e pelos cavaleiros. Acupuntura reduziu significativamente a razão $\mathrm{LF} / \mathrm{HF}$ ( $\mathrm{p}<0,0001$ ) um índice que indica o balanço simpato-vagal sugerindo que a acupuntura pode reduzir a resposta autonômica cardíaca em situação de estresse. No entanto, a acupuntura não teve efeito significativo sobre a frequência cardíaca, sobre os níveis de cortisol e sobre o desempenho avaliado pelos juízes e cavaleiros. Nossos resultados sugerem que a acupuntura pode reduzir algumas respostas de estresse em cavalos atletas submetidos a provas de reprise de adestramento e que este efeito se deva a sua ação potencializadora da atividade vagal.

Palavras-chave: bem-estar animal, cortisol, variabilidade da freqüência cardíaca, comportamento, esporte.

\begin{abstract}
Horses have a natural predisposition for sport, however, their use in competitions can result in stress-related problems that impair their sporting performance and especially their health. Thus, the use of strategies that can prevent or minimize the deleterious effects of stress is essential. In this sense, acupuncture is an ancient technique of Traditional Chinese Medicine that has been used in the treatment and prevention of stress-related diseases. Therefore, we evaluated whether acupuncture can alter stress responses in horses submitted to a dressage test. Six Brasileiro de Hipismo horses, aged between 6-8 years old, used to dressage training, from Army Riding School, Rio de Janeiro, Brazil were used. The effect of acupuncture at points GV1, HT7, GV2O, and B52 immediately before to the dressage was evaluated through analysis of test performance, serum cortisol and heart rate variability (HRV). Polar Equine ${ }^{\mathrm{TM}}$ heart rate monitor was used for HRV analysis and blood samples were collected before and 30 minutes after the end of the dressage. The performance was judged by official judges and by the riders. Acupuncture significantly reduced the LF / HF ratio $(\mathrm{p}<0.0001)$, an index that indicates the sympathovagal balance suggesting that acupuncture can reduce the cardiac autonomic response in stressful situations. However, acupuncture
\end{abstract}

Como citar: Villas-Boas, J. D., Almeida, N. A. S. Almeida, F. Q., \& Medeiros, M. A. (2017). Efeito da acupunturanas respostas de estresse em equinos atletas submetidos a reprise de adestramento. Brazilian Journal of Veterinary Medicine, 39(4), 221-230. doi: 10.29374/2527-2179.bjvm020517

Fonte de financiamento: CAPES

Conflito de interesses: Os autores declaram não haver conflito de interesses que precisam ser informados.

Recebido: Outubro 17, 2017

Aceito: Dezembro 22, 2017

O estudo foi realizado na Escola de Equitação do Exército (ESEQEX), Rio de Janeiro, RJ, Brasil.

\section{${ }^{*}$ Correspondência}

Magda Alves de Medeiros

Programa de Pós-graduação em Medicina Veterinária, Universidade Federal Rural do Rio de Janeiro - UFRRJ

Rod. BR 465, Km 7, Campus Universitário CEP 23890-000 - Seropédica (RJ), Brasil E-mail: magda.medeiros@gmail.com
Copyright Villas-Boas et al. Este é um artigo publicado em acesso aberto (Open Access) sob a licença Creative Commons Attribution Non-Commercial, que permite uso, distribuiç̧ão e reprodução em qualquer meio, sem restrições desde que sem fins comerciais e que o trabalho original seja corretamente citado. 
had no effect on heart rate, cortisol levels and performance assessed by judges and riders. Our results suggest that acupuncture may reduce some stress responses in horse athletes submitted to dressage and that this effect can be related to its potentiating action of vagal activity.

Keywords: animal welfare, cortisol, heart rate variability, behavior, sport.

\section{Introdução}

O cavalo tem uma predisposição natural para o esporte, no entanto, o seu uso em competições frequentemente resulta em problemas relacionados ao estresse que direta ou indiretamente influenciam seu desempenho esportivo, bem-estar e produzem efeitos deletérios a sua saúde.

A resposta ao estresse é iniciada quando um estímulo (o estressor) é percebido como uma ameaça potencial, resultando numa combinação de respostas biológicas projetadas para aliviar os efeitos do estressor percebido. Em situações de estresse, ocorre a ativação de duas principais vias: o eixo hipotálamo-hipófise-adrenal (HHA), levando ao aumento da produção de cortisol e o sistema nervoso simpático, promovendo a liberação de catecolaminas (noradrenalina/ adrenalina). Apesar desta resposta ser fundamental para a sobrevivência do indivíduo, se a ativação autonômica e a liberação de cortisol for exagerada pode levar a imunidade reduzida e aumentar o risco de doenças como hipertensão, doenças cardíacas, fadiga e insônia (Teixeira et al., 2015; McEwen, 2007). Segundo, Devries et al. (2007), distúrbios de comportamento e estresse são umas das principais causas de doenças, lesões e morte em equinos (DeVries et al., 2007).

Cayado et al. (2006) afirmam que competições em diversas modalidades, provocam uma clássica resposta fisiológica de estresse, sugerindo que cavalos envolvidos em competição podem proporcionar um modelo de estresse induzido pelo exercício (Cayado et al., 2006). O adestramento ou dressage éuma das três modalidades olímpicas, regulada pela Federação Equestre Internacional (FEI), onde o cavalo e o cavaleiro devem realizar uma série de movimentos predeterminados. Nas competições os juízes avaliam cada movimento realizado com base num padrão objetivo predeterminado e atribuem pontuações. Numa reprise (prova de adestramento) o cavalo não deve parecer nervoso, andar torto ou rígido. Para que ocorra um movimento harmônico do conjunto cavaleiro-cavalo, o cavalo éimpedido a expressar suas emoções e há uma considerável restrição física nos movimentos do animal. Hausberger et al. (2009) demonstrou que cavalos de adestramento, comparado a outras modalidades equestres como volteio e salto, apresentam alto índices de estereotipias sugerindo que estes animais são submetidos a níveis de estresse mais altos que animais de outras modalidades (Hausberger et al., 2009). Desta forma, uma prova de reprise adestramento pode representar um estímulo estressor e pode ser usada como modelo estresse para avaliar a eficiência de estratégias anti-estresse.

Em vista da necessidade de melhorar a qualidade de vida e o bem-estar animal (equilíbrio físico e mental) e consequentemente melhorar o desempenho atlético é fundamental o desenvolvimento de estratégias para minimizar os efeitos do estresse. Desta forma, cada vez mais tem se buscado alternativas terapêuticas, não-farmacológicas para diminuir as consequências deletérias das respostas de estresse. Os medicamentos convencionalmente utilizados para reduzir as respostas de estresse produzem diversos efeitos colaterais indesejados e no cavalo atleta o uso de medicamentos deve ser avaliado com cautela porque pode ser considerado dopping. Nesse sentido, estudos sobre os efeitos da acupuntura e sua aplicação clínica no tratamento e prevenção do estresse são de interesse na medicina equestre.

A acupuntura é uma técnica terapêutica milenar da Medicina Tradicional Chinesa, que ao longo dos anos vem sendo difundida no Ocidente. A acupuntura tem como princípio básico a estimulação de pontos específicos no corpo para gerar um efeito terapêutico ou homeostático (Xie 2007). Na medicina ocidental contemporânea, a acupuntura é considerada uma forma de estimulação sensorial que afeta várias classes de fibras nervosas aferentes e uma vez direcionado para a medula espinhal e os centros superiores do SNC, desencadeia os eventos neurofisiológicos que geram a resultado terapêutico (Soligo et al., 2013). Diferentes mecanismos neurobiológicos tem sido propostos para os efeitos analgésicos (Han 2003, anti-inflamatórios (McDonald et al., 2015) e anti-estresse (Eshkevari et al., 2013; Kondo et al., 2014) da acupuntura.

Embora a acupuntura tenha sido usada para tratar várias condições em cavalos, a literatura é escassa em estudos relativos aos efeitos da acupuntura em condições relacionadas ao estresse em cavalos. No presente estudo, testamos a hipótese de que uma única sessão de acupuntura 
imediatamente antes de uma prova de reprise de adestramento poderia tranquilizar o animal, produzindo mudanças autonômicos, endócrinas e comportamentais, que pudessem de alguma formar melhorar o bem-estar, o desempenho e a recuperação desses atletas.

\section{Materiais e métodos}

\section{Animais}

Foram utilizados 6 equinos, machos e fêmeas, com idade entre 6 e 8 anos, da raça Brasileiro de Hipismo pesando entre 450-550 kg, em condições saudáveis, sem sintomas de doenças, provenientes da Escola de Equitação do Exército. Todos os cavalos foram treinados continuamente desde os 5 anos de idade e seguiram uma rotina de treinamento de 6 dias por semana, incluindo exercícios de galope, salto e adestramento. Os cavalos eram alojados em baias de alvenaria individuais de $4 \times 4 \mathrm{~m}$, com depósito de água e comida. As portas das baias permitiam o contato visual entre os cavalos. Os cavalos eram alimentados com concentrado coast-cross e tiveram acesso gratuito à água da torneira.

Todos os procedimentos experimentais foram aprovados pelo Comitê de Pesquisa e Ética Animal e Humana da Universidade Rural Federal do Rio de Janeiro / COMEP-UFRRJ / Brasil (protocolo no 230833.002064 / 2012-10).

\section{Protocolo experimental}

O experimento foi realizado em dois dias, com intervalo de uma semana. No dia 1, os animais foram submetidos a prova de reprise de adestramento sem tratamento prévio (grupo CTL) e no dia 2, os mesmos animais foram tratados com acupuntura antes da prova (grupo ACUP). Iniciando-se as 9:OOh, com cada animal em sua respectivas baia, a fita com eletrodos do frequencímetro cardíaco Polar Equine ${ }^{\circledR}$ ao redor do toráx foi colocada e ativada, e foram realizadas as coletas de sangue para análise de cortisol basal. No dia 1, logo após a colocação da fita e a coleta de sangue, os animais foram encilhados e ficaram pelo período de 20 minutos esperando em suas baias, estacados, sem qualquer tipo de manipulação. Após esse período, os animais foram conduzidos para a área ao redor do picadeiro para o aquecimento por 10 minutos. Em seguida, foram conduzidos ao picadeiro onde foram submetidos a uma Reprise de Adestramento. No dia 2, nos mesmos animais, após o encilhamento os animais foram submetidos ao tratamento de acupuntura, que consistiu na aplicação de agulhas de aço inox 0,30 × $40 \mathrm{~mm}$, marca Dongbang com a profundidade de $2 \mathrm{~cm}$ nos pontos VG1, C7, VG20 e B52. Após 20 minutos sem qualquer manipulação, as agulhas foram retiradas e os animais foram conduzidos para a área ao redor do picadeiro para o aquecimento de 10 minutos. Ao término desse período, os cavalos foram conduzidos ao picadeiro onde foram submetidos a uma Reprise de Adestramento.

A Reprise teve duração de aproximadamente 6 minutos. No momento da reprise os animais foram avaliados por dois juízes oficiais (credenciados pela Confederação Brasileira de Hipismo)e pelos seus respectivos cavaleiros, que não sabiam em que grupo o animal pertencia. Ao término da reprise, os animais foram levados até suas baias, onde foram desencilhados. Após 30 minutos do término da reprise, foi realizada a coleta de sangue para análise de cortisol sérico e retirada a fita com os eletrodos do frequencímetro cardíaco.

\section{Tratamentos}

- Controle: no dia 1 os animais permaneceram por 20 minutos sem qualquer manipulação ( $n=6)$;

- Acupuntura: no dia 2, os mesmos animais receberam agulhas de metal 0,30 × 40 mm, marca Dongbang com a profundidade de aproximadamente $2 \mathrm{~cm}$ nos pontos VG1, C7, VG2O e B52 por 20 minutos imediatamente antes da reprise de adestramento (n=6). A localização dos pontos está descrita nas Figura 1 e Tabela 1.

\section{Análise da variabilidade da frequência cardíaca}

Para a análise da variabilidade da frequência cardíaca (VFC) foram selecionados os momentos basais, 15 minutos e 30 minutos após a reprise e análise da variabilidade da frequência cardíaca foi realizada utilizando-se programa personalizado (CardioSeries v2.4). Os valores do intervalo 


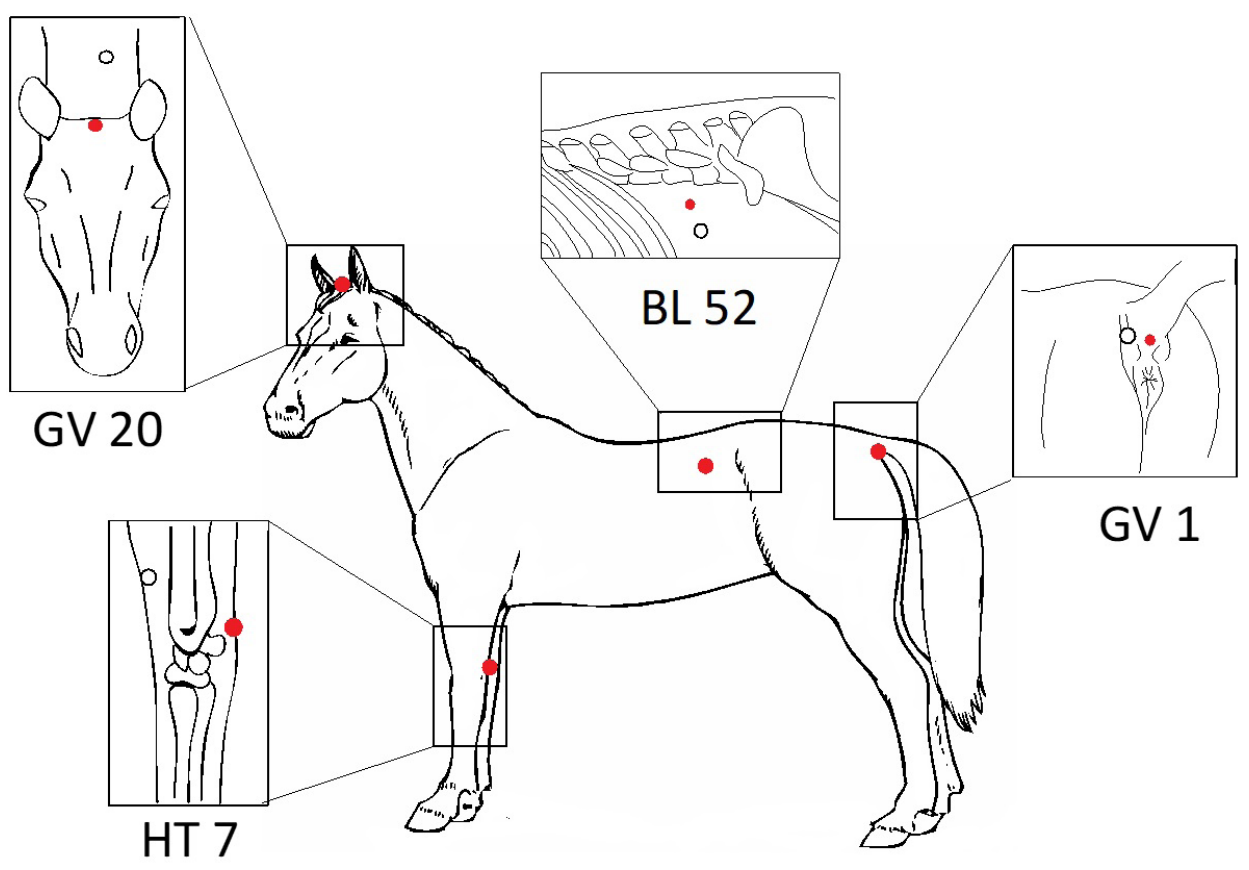

Figura 1. Alterar o texto para: Localização dos pontos VC2O, C7, B52 e VG1 (em ingles: GV20, HT7, BL52, VG1 respectivamente) em equinos.

Fonte: Villas-Boas et al. (2016).

Tabela 1. Localização e indicação dos pontos de acupuntura utilizados.

\begin{tabular}{|c|c|c|}
\hline Acuponto & Localização & Indicação Tradicional \\
\hline $\begin{array}{l}\text { VG-1 } \\
\text { (HouHai) } \\
\text { Mar de Trás }\end{array}$ & $\begin{array}{l}\text { Ponto médio entre a cauda e o ânus, na prega } \\
\text { anocaudal. }\end{array}$ & $\begin{array}{l}\text { Nervosismo, agitação e } \\
\text { acalma a mente; Alterações } \\
\text { na porção final do intestino } \\
\text { grosso }\end{array}$ \\
\hline $\begin{array}{l}\text { VG20 } \\
\text { (Bai Hui) } \\
\text { Os Cem encontros }\end{array}$ & $\begin{array}{l}\text { Na linha média, no ponto mais alto da fronte, rostral } \\
\text { a crista do occipital }\end{array}$ & $\begin{array}{l}\text { Reunião dos meridianos } \\
\text { Yang, acalma a mente }\end{array}$ \\
\hline $\begin{array}{l}\text { C7 } \\
\text { (ShenMen) } \\
\text { Portão do Espírito }\end{array}$ & $\begin{array}{l}\text { A localização superficial é numa depressão na } \\
\text { superfície medial do rádio, cranial a inserção do } \\
\text { músculo flexor ulnar do carpo, ao nível do aspecto } \\
\text { dorsal do músculo acessório do carpo. A localização } \\
\text { mais profunda é entre os músculos flexor ulnar do } \\
\text { carpo e o flexor digital superficial. }\end{array}$ & $\begin{array}{l}\text { Acalma o Espírito, regula } \\
\text { a pressão arterial; O } \\
\text { coração é responsável pelo } \\
\text { equilíbrio emocional }\end{array}$ \\
\hline $\begin{array}{l}\text { B52 } \\
\text { (Zhishi) } \\
\text { Morada da Vontade }\end{array}$ & $\begin{array}{l}\text { Localizado a } 6 \text { cun (unidade de medida relativa } \\
\text { na utilizada na MTC, onde } 1 \text { cun equivale, no caso } \\
\text { dos equinos, à largura da costela flutuante à linha } \\
\text { média dorsal, na altura dos processos espinhosos } \\
\text { da } 2^{\text {a e }} 3^{\mathrm{a}} \text { vértebras lombares }\end{array}$ & $\begin{array}{l}\text { Tonifica a Essência, } \\
\text { beneficia a região lombar; } \\
\text { Controla o medo; Reforça a } \\
\text { vontade }\end{array}$ \\
\hline
\end{tabular}

Fonte: Shoen (2001), Xie e Priest (2007).

RR dos diferentes momentos obtidos pelo programa do Polar Pro-Trainer foram exportados em arquivo de texto (TXT) para o programa CardioSeries v2.4 que calcula parâmetros VFC no domínio da frequência e no domínio do tempo. Segmentos de 64 s foram analisados utilizando a Transformada Rápida de Fourier (FFT) a partir da qual se obteve os valores dos componentes frequências de baixa frequência (LF), alta frequência (HF) e a razão entre LF e HF. Essa razão permite identificar o balanço entre a ativação simpática e parassimpática nos momentos analisados sendo um importanteíndice de ativação autonômica cardíaca. Os valores das variáveis utilizadas 
pelo programa CardioSeries foram padronizados da seguinte forma: LF 0,01 - 0,07; HF - 0,070,5; taxa de interpolação = 4 e pontos no domínio da freqüência = 256 (Villas-Boas et al., 2015; Villas-Boas et al., 2016).

\section{Análise de cortisol}

As coletas foram realizadas nos momentos basal e 30 minutos após a reprise. Amostras de sangue da veia jugular foram coletados em tubos SST Vacutainer. Após a coleta, o sangue foi centrifugado durante 10 minutos a 3200 rpm. O soro ( $3 \mathrm{~mL})$ foi coletado em tubos de plástico e manteve a $-20{ }^{\circ} \mathrm{C}$. As concentrações séricas de cortisol foram determinadas, em duplicado, por meio de um método de radioimunoensaio de duplo anticorpo usando um kit comercial (RD Coated Tube Cortisol I125 RIA, Costa Mesa, CA, EUA). A sensibilidade do ensaio foi 0,17 $\mu \mathrm{g} / \mathrm{dL}$ e o coeficiente de variação intra-ensaio foi 6,59\%.

\section{Avaliação dos juízes}

Dois juízes foram posicionados ao longo do lado menor do picadeiro, a uma distância de 2 metros do cercado. Todos os movimentos e determinadas transições, que foram julgados pelos juízes. A avaliação dos juízes consistiu na avaliação da técnica a qual foi realizada durante a reprise e na avaliação do conjunto, a qual foi realizada logo após a reprise.

Os animais receberam graus de 0 a 10 dados por cada juiz, de acordo com a seguinte correlação. (10 - Excelente; 9 - Muito Bom; 8 - Bom; 7 - Quase bom; 6 - Satisfatório; 5 - Suficiente; 4 - Insuficiente; 3 - Quase mal; 2 - Mal; 1 - Muito mal; 0 - Não executado).

\section{Avaliação dos cavaleiros}

Logo após a reprise, os cavaleiros receberam um questionário no qual os cavalos foram avaliados quanto à flexibilidade, relaxamento, concentração/atenção, aceitação/obediência e vontade. A pontuação variou de 0 a 10 em cada item.

\section{Análises estatísticas}

As variáveis quantitativas (VFC e Cortisol) foram submetidas à Anova de duas vias para medidas repetidas (fatores tempo, dia/tratamento e interação), quando significativas foram comparadas pelo Teste de Bonferroni, a 5\% de probabilidade. Para análise do desempenho foi utilizada o test t pareado. As análises foram realizadas e os gráficos construídos através do programa GraphPad Prism 6.

\section{Resultados}

Na análise da VFC, a ANOVA de duas vias para medidas repetidas detectou diferença significativa no fator tempo (sem considerar efeito dia/tratamento), mostrando que reprise de adestramento do foi capaz de aumentar a razão LF/HF ( $\left.F_{(1,20)}=53,50, p<0,0001\right), \operatorname{LF}\left(F_{(1,20)}=26,71, p<0,0001\right)$ e frequência cardíaca $\left(F_{(1,20)}=25,46, p<, 0001\right)$ e de diminuir a $\operatorname{HF}\left(F_{(1,20)}=21,26, p<0,0001\right)$. Quando avaliamos o efeito do dia/tratamento (sem considerar efeito tempo), a ANOVA de duas vias para medidas repetidas detectou diferença significativa na razão $\operatorname{LF} / \mathrm{HF}\left(\mathrm{F}_{(1,10)}=15,62 \mathrm{p}<0,002\right)$. No fator interação, a ANOVA de duas vias para medidas repetidas detectou diferença significativa nas bandas de frequência $\operatorname{LF}\left(\mathrm{F}_{(2,20)}=5,11, \mathrm{p}=0,016\right)$ e HF $\left(\mathrm{F}_{(2,20)}=4,44, \mathrm{p}=0,025\right)$ e na razão $\mathrm{LF} / \mathrm{HF}$ $\left(\mathrm{F}_{(2,20)}=16,42, \mathrm{p}<0,0001\right)$. O teste de comparações múltiplas Bonferroni foi capaz de identificar diferenças entre o grupo ACUP eCTL $(p<0,05)$ no momento 15 minutos após a reprise, onde o grupo ACUP apresentou valores de LF/HF e LF significativamente menores e de HF significativamente maiores que o CTL, indicando que a acupuntura foi capaz de reduzir o aumento de LF e de LF/HF e a redução de HF induzida pela reprise. No entanto, não foi detectada diferença significativa na frequência cardíaca entre os grupos (Figura 2).

Na análise dos níveis de cortisol sérico, a ANOVA de duas vias para medidas repetidas detectou diferença no fator dia/tratamento $\left(\mathrm{F}_{(1,10)}=5,22 ; \mathrm{p}=0,0453\right)$, sem detectar diferença significativa nos fatores tempo $\left(F_{(1,10)}=3,514 ; p=0,09\right)$ e interação $\left(F_{(1,10)}=0,016 ; p=0,90\right)$. O que indica que a reprise não produziu aumento significativo nos níveis de cortisol e que no segundo dia de teste 
os valores de cortisol estavam menores que o primeiro dia de teste tanto nos momentos basal como após a reprise (Figura 3).

O teste de t pareado não detectou diferença significativa no desempenho dos animais ao serem tratados com acupuntura, tanto na avaliação técnica quanto na avaliação do conjunto feitas pelos juízes oficias, assim como na avaliação realizada pelos cavaleiros (Tabela 2).
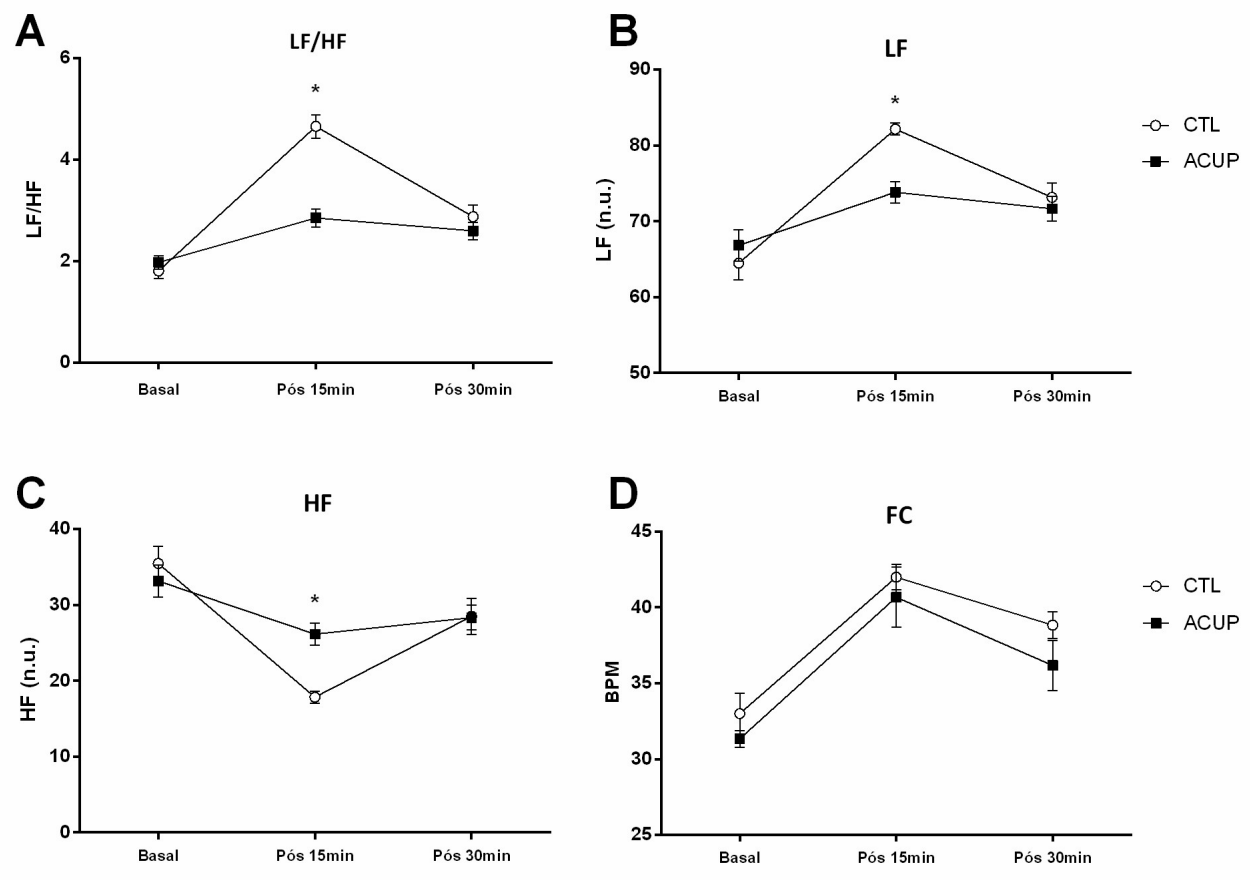

Figura 2. Efeito da acupuntura na Variabilidade da Frequência Cardíaca de equinos submetidos a reprise de adestramento. Os dados são apresentados como média \pm epm nos momentos Basal, 15 e 30 minutos após o exercício. Antes os equinos foram tratados com acupuntura por 20 min imediatamente antes da reprise nos pontos VG1, VG2O, C7 e B52 (ACUP) e não tratados (CTL). (A) razão LF/HF; (B) componente de baixa frequência LF; (C) componente de alta frequência HF; e (D) Frequência Cardíaca Média. * indica diferença significativa entre os ACUP e CTL $(\mathrm{p}<0,05)$.

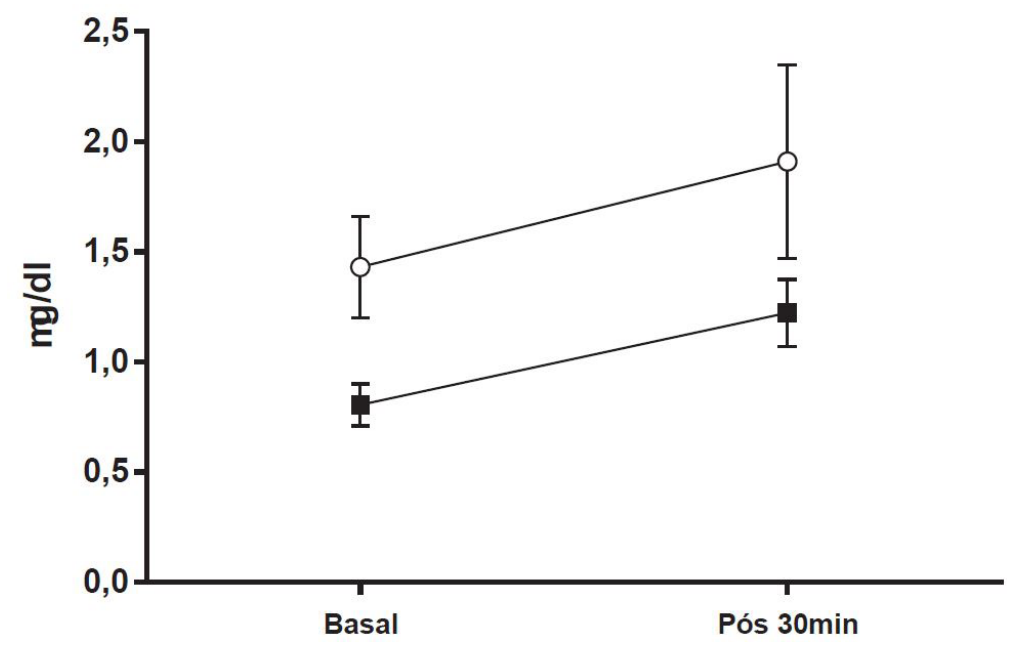

Figura 3. Efeito da acupuntura nos níveis de Cortisol Sérico de equinos submetidos a reprise de Adestramento. Cortisol sérico em $\mu \mathrm{g} / \mathrm{dl}$, antes (basal), e 30 min após a reprise. Os equinos foram previamente tratados por $20 \mathrm{~min}$ com acupuntura nos pontos VG1, VG2O, C7 e B52 (ACUP) e não tratados (CTL). 
Tabela 2. Desempenho dos animais segundo os juízes (avaliação técnica e do conjunto) e os cavaleiros (dados apresentados como média $\pm \mathrm{dp}$ ).

\begin{tabular}{ccc}
\hline & Dia 1: CTL & Dia 2: ACUP \\
\hline Avaliação técnica dos juízes & $160,4 \pm 4,3$ & $163,2 \pm 4,7$ \\
Avaliação conjunto dos juízes & $36,5 \pm 2,0$ & $36,2 \pm 1,7$ \\
Avaliação dos cavaleiros & $30,33 \pm 5,64$ & $30,33 \pm 4,68$ \\
\hline
\end{tabular}

A avaliação dos juízes seguiu o modelo padrão da CBH e na avaliação do cavaleiros foi considerada flexibilidade, relaxamento, concentração, aceitação e vontade do animal.

\section{Discussão}

Exercício físico representa um desafio a homeostase. Eapesar do seu indubitável efeito benéfico sobre a saúde, o exercício representa um estímulo de estresse, uma vez que o organismo altera muitos sistemas para se adaptar a uma nova condição (Cayado et al., 2006). Reconhece-se que a quantidade de tensão psicológica que o animal encontra-se determina o grau de resposta do eixo HPA. Em atletas, o estresse emocional da competição é um elemento importante na resposta adrenal (Suinn, 2005). Cayado et al. (2006) sugerem que cavalos envolvidos em competição são um bom modelo para o estudo da resposta ao estresse induzida pelo exercício e que esse modelo pode ser utilizado em pesquisas em busca de novos fármacos ansiolíticos ou mesmo conduzir à descoberta de moléculas endógenas ansiolíticas em cavalos. No adestramento, e em especial em provas de reprise, o animal precisa estar flexível, calmo, atento, o que sugere que o estresse influencia mais o desempenho atlético do cavalo de adestramento do que de outras modalidades. Desta forma, as provas de reprise podem ser bons modelos de estresse físico e psicológico em cavalos.

Nossos resultados mostram que a reprise alterou os parâmetros de VFC, levando a um aumento do componente de alta frequência (LF), da razão entre os componentes de alta e baixa frequencia (razão LF/HF) e da frequencia cardíaca (FC) e diminuição do componente de baixa frequencia (HF). Indicando um aumento da atividade simpática e redução da atividade parassimpática, característica de situações de estresse. Neste sentido, vale ressaltar que a análise da VFC se mostra uma ferramenta muito sensível na análise das respostas de estresse em equinos. Apesar dos níveis de cortisol apresentarem aumento após a reprise, este aumento não foi estatisticamente significativo. Estes resultados podem estar relacionados a grande variabilidade dos níveis de cortisol em condições basais e em resposta de estresse moderado, o que leva a entender que a interpretação dos níveis de cortisol em situações de estresse são complexas e estão sendo repensadas por estudos atuais (Pawluski et al., 2017).

Nossos resultados também mostram que quando os animais receberam a estimulação nos pontos de acupuntura VG1, C7, VG2O e B52, houve uma redução da banda LF e da razão LF/HF e um aumento do HF 15 minutos após a reprise de adestramento. Não foi observado nenhum efeito da acupuntura na frequência cardíaca. Estes resultados indicam um efeito notável da acupuntura sobre o equilíbrio simpatovagal. A diminuição da razão LF / HF pode ser atribuído a um aumento simultâneo do tônus vagal e diminuição do tônus simpático. Recentemente, vários estudos abordaram o efeito autonômico da estimulação de pontos de acupuntura utilizando a análise da VFC (Villas-Boas et al., 2015; Li et al., 2013; Lee et al., 2010; Anderson et al., 2012). Embora alguns estudos demonstraram que a estimulação de alguns acupontos pode aumentar a razão LF / HF (Imai et al., 2008), outros estudos mostraram que acupuntura pode reduzir a relação LF/HF, tanto aumentando a potência HF (alta freqüência) ou diminuindo o LF (baixa freqüência) (Anderson et al., 2012; Villas-Boas et al., 2015). O efeito da acupuntura na VFC dependerá principalmente da condição do animal (por exemplo, saudável, doente, ou sob estresse) e os parâmetros da acupuntura (i.e., acupontos escolhidos e tipo de estimulação) empregados (Chung et al., 2014). Muitos estudos mostram os efeitos positivos da estimulação dos pontos de acupuntura para produzir sedação e acalmar (Hwang et al., 2011).

Em relação ao efeito da acupuntura sobre os níveis de cortisol, nossos resultados mostram que como foi detectado efeito apenas no fator dia/tratamento e não na interação, isso significa que no segundo dia de teste os valores de cortisol estavam menores que o primeiro dia de teste tanto 
nos momentos basal como após a reprise (Figura 3). Ou seja, como a estimulação de pontos foi realizada após a coleta basal, não podemos afirmar esta diferença entre o primeiro e o segundo dia esteja relacionada ao efeito da acupuntura. Este efeito da acupuntura nos níveis de cortisol deve ser confirmado em estudos posteriores. Outros estudos também não conseguiram mostrar redução ou inibição da elevação dos níveis de cortisol induzida pelo estresse (Angeli \& Luna, 2008; Godoi et al., 2014). Por outro lado, alguns estudos mostraram o efeito da acupuntura na prevenção do aumento dos níveis de cortisol após modelo experimental de sobressalto (VillasBoas et al., 2016) e após transporte rodoviário e exercício físico (Rizzo et al., 2017b). O mecanismo de ação da acupuntura tem sido associado ao bloqueio da ativação HPA induzida pelo estresse (Eshkevari et al., 2013; Lee et al., 2004). Este mecanismo pode explicar, pelo menos em parte, o efeito da acupuntura na prevenção dos efeitos deletérios do estresse.

No presente estudo, não houve alteração do desempenho de equinos ao serem tratados com acupuntura. Nesta prova existe uma interferência muito grande do cavaleiro, tanto o cavalo quanto o cavaleiro devem estar confiantes e entrosados para efetuar os movimentos predeterminados. Assim, qualquer pequeno erro do cavaleiro, a qualidade do movimento fica comprometida prejudicando a pontuação.

Não existem pesquisas anteriores sobre o efeito da acupuntura no desempenho de equinos na modalidade Adestramento e a literatura sobre os efeitos da estimulação de acupuntos em cavalos é escassa. Portanto, os acupontos utilizados neste estudo foram escolhidos baseados na indicação pela MTC para tratar distúrbios emocionais em cavalos (Schoen, 2001). Vale ressaltar que os resultados obtidos foram a partir de apenas uma sessão de acupuntura imediatamente antes da reprise. Erlich \& Haber (1992) demonstraram aumento significativo na capacidade atlética de atletas humanos tratados com sessões semanais de acupuntura, durante 5 semanas. Karvelas et al. (1996) não encontraram diferença nos parâmetros fisiológicos em humanos após único tratamento com acupuntura. Estes trabalhos sugerem a necessidade de fazer-se mais de um tratamento para que haja alguma resposta de melhora fisiológica de melhora de performance de atletas humanos. Em geral, os estudos que utilizam protocolos que se assemelham à prática clínica usual são mais propensos a obter melhores resultados (Anderson et al., 2012). De fato, o uso clínico da acupuntura normalmente inclui 4 a 10 pontos de acupuntura (ou até mais) dados em cada uma das seis (ou mesmo mais) sessões, seguindo um protocolo individualizado de pontos após um diagnóstico pela MTC. No entanto, uma grande parte das pesquisas científicas com acupuntura realizadas tanto em animais quanto em humanos (Yang et al., 2002; Akimoto et al., 2003; Angeli \& Luna, 2008; Godoi et al., 2014; Rizzo et al., 2017a) utilizam um protocolo único e padronizado de tratamento, e não um protocolo individualizado. Quanto se utiliza protocolos padronizados é possível avaliar o efeito específico desses pontos no tratamento de uma determinada patologia. No entanto, a desvantagem do utilização de um protocolo único e padronizado é que o indivíduo não é tratado de forma completa, ou seja, outros problemas que possam estar influenciando ou o desequilíbrio desse problema, o que pela MTC é conhecido como "raiz", podem não estar sendo tratados, podendo prejudicar de certa forma o resultado.

Portanto, o fato de no presente estudo não ter sido observado melhora significativa em alguns dos parâmetros analisados pode ser devido a utilização de um protocolo único de tratamento. Dessa forma, se faz necessário mais estudos com protocolos diferentes ou individualizados e/ou a utilização de outras técnicas para melhor avaliar o efeito da acupuntura em diversas condições estressantes no cavalo atlético.

\section{Conclusões}

Apenas uma sessão de acupuntura nos pontos VG1, VG2O, C7 e B52 imediatamente antes da reprise de adestramento reduziu a razão $\mathrm{LF} / \mathrm{HF}$, indicando que a acupuntura promove regulação autonômica, provavelmente pelo aumento da atividade vagal. Apesar de não ter sido detectado efeito da acupuntura nos níveis de cortisol e no desempenho atlético, nossos resultados sugerem que a acupuntura pode ser uma terapia promissora no controle do estresse em equinos. Mas ainda se faz necessário mais estudos com diferentes protocolos de estimulação de pontos para elucidar a eficácia e os mecanismos fisiológicos da acupuntura na nas respostas de estresse em equinos. 


\section{Referências}

Akimoto, T., Nakahori, C., Aizawa, K., Kimura, F., Fukubayashi, T., \& Kono, I. (2003). Acupuncture, and responses of immunologic and endocrine markers during competition. Medicine and Science in Sports and Exercise, 35(8), 1296-1302. http://dx.doi.org/10.1249/01.MSS.0000078934.07213.25. PMid:12900681.

Anderson, B., Nielsen, A., McKee, D., Jeffres, A., \& Kligler, B. (2012). Acupuncture and heart rate variability: a systems level approach to understanding mechanism. Explore, 8(2), 99-106. http://dx.doi.org/10.1016/j. explore.2011.12.002. PMid:22385564.

Angeli, A. L., \& Luna, S. P. L. (2008). Aquapuncture improves metabolic capacity in thoroughbred horses. Journal of Equine Veterinary Science, 28(9), 525-531. http://dx.doi.org/10.1016/j.jevs.2008.07.023.

Cayado, P., Muñoz-Escassi, B., Domínguez, C., Manley, W., Olabarri, B., Muela, M. S., Castejon, F., Marañon, G., \& Vara, E. (2006). Hormone response to training and competition in athletic horses. Equine Veterinary Journal. Supplement, 36(36), 274-278. http://dx.doi.org/10.1111/j.2042-3306.2006.tb05552.x. PMid:17402431.

Chung, J. W. Y., Chung, V. C. M., Yan, A., \& Zhang, H. (2014). Effect of acupuncture on heart rate variability: a systematic review. Evidence-Based Complementary and Alternative Medicine, 2014, 19. http://dx.doi. org/10.1155/2014/819871. PMid:24693326.

DeVries, A. C. K. S., Craft, T. K. S., Glasper, E. R., Neigh, G. N., \& Alexander, J. K. (2007). Social influences on stress responses and health. Psychoneuroendocrinology, 32(6), 587-603. PMid:17590276.

Ehrlich, D., \& Haber, P. (1992). Influence of acupuncture on physical performance capacity and haemodynamic parameters. International Journal of Sports Medicine, 13(6), 486-491. http://dx.doi.org/10.1055/s-2007-1021303. PMid:1428381.

Eshkevari, L., Permaul, E., \& Mulroney, S. E. (2013). Acupuncture blocks cold stress-induced increases in the hypothalamus-pituitary-adrenal axis in the rat. The Journal of Endocrinology, 217(1), 95-104. http://dx.doi. org/10.1530/JOE-12-0404. PMid:23386059.

Godoi, T. L. O., Villas-Boas, J. D., Almeida, N. A. D. S., Trigo, P. I., Almeida, F. Q., \& Medeiros, M. A. (2014). Pharmacopuncture versus acepromazine in stress responses of horses during road transport. Journal of Equine Veterinary Science, 34(2), 294-301. http://dx.doi.org/10.1016/j.jevs.2013.06.008.

Han, J.-S. (2003). Acupuncture: neuropeptide release produced by electrical stimulation of different frequencies. Trends in Neurosciences, 26(1), 17-22. http://dx.doi.org/10.1016/S0166-2236(02)00006-1. PMid:12495858.

Hausberger, M., Gautier, E., Biquand, V., Lunel, C., \& Jégo, P. (2009). Could work be a source of behavioural disorders? A study in horses. PLoS One, 4(10), e7625. http://dx.doi.org/10.1371/journal.pone.0007625. PMid:19862328.

Hwang, D.-S., Kim, H. K., Seo, J. C., Shin, I. H., Kim, D. H., \& Kim, Y.-S. (2011). Sympathomodulatory effects of Saam acupuncture on heart rate variability in night-shift-working nurses. Complementary Therapies in Medicine, 19(Suppl 1), S33-S40. http://dx.doi.org/10.1016/j.ctim.2010.11.001. PMid:21195293.

Imai, K., Ariga, H., Chen, C., Mantyh, C., Pappas, T. N., \& Takahashi, T. (2008). Effects of electroacupuncture on gastric motility and heart rate variability in conscious rats. Autonomic Neuroscience: Basic and Clinical, 138(12), 91-98. http://dx.doi.org/10.1016/j.autneu.2007.11.003. PMid:18083640.

Karvelas, B. R., Hoffman, M. D., \& Zeni, A. L. (1996). Acute effects of acupuncture on physiological and psychological responses to cycle ergometry. Archives of Physical Medicine and Rehabilitation, 77(12), 1256-1259. http://dx.doi. org/10.1016/S0003-9993(96)90189-6. PMid:8976308.

Kondo, T., \& Kawamoto, M. (2014). Acupuncture and moxibustion for stress-related disorders. BioPsychoSocial Medicine, 8(1), 7. http://dx.doi.org/10.1186/1751-0759-8-7. PMid:24456818.

Lee, H.-J., Lee, B., Choi, S.-H., Hahm, D.-H., Kim, M.-R., Roh, P.-U., Pyun, K.-H., Golden, G., Yang, C.-H., \& Shim, I. (2004). Electroacupuncture reduces stress-induced expression of c-Fos in the brain of the rat. The American Journal of Chinese Medicine, 32(5), 795-806. http://dx.doi.org/10.1142/S0192415X04002405. PMid:15633814.

Lee, S., Lee, M. S., Choi, J.Y., Lee, S.-W., Jeong, S.-Y., \& Ernst, E. (2010). Acupuncture and heart rate variability: a systematic review. Autonomic Neuroscience: Basic and Clinical, 155(1-2), 5-13. http://dx.doi.org/10.1016/j. autneu.2010.02.003. PMid:20304708.

Li, Q. Q., Li, G. X., Shi, Q., Xu, J., Wang, C. Z., Liu, A., \& Wang, L. P. (2013). Acupuncture effect and central autonomic regulation. Evidence-Based Complementary and Alternative Medicine, 2013, 6. PMid:23762116.

McDonald, J. L., Cripps, A. W., \& Smith, P. K. (2015). Mediators, receptors, and signalling pathways in the antiinflammatory and antihyperalgesic effects of acupuncture. Evidence-Based Complementary and Alternative Medicine, 2015, 1. http://dx.doi.org/10.1155/2015/975632. PMid:26339274.

McEwen, B. S. (2007). Physiology and neurobiology of stress and adaptation: central role of the brain. Physiological Reviews, 87(3), 873-904. http://dx.doi.org/10.1152/physrev.00041.2006. PMid:17615391.

Pawluski, J., Jego, P., Henry, S., Bruchet, A., Palme, R., Coste, C., \& Hausberger, M. (2017). Low plasma cortisol and fecal cortisol metabolite measures as indicators of compromised welfare in domestic horses (Equus caballus). PLoS One, 12(9), e0182257. http://dx.doi.org/10.1371/journal.pone.0182257. PMid:28886020.

Rizzo, M., Arfuso, F., Giannetto, C., Giudice, E., Longo, F., Bruschetta, D., \& Piccione, G. (2017a). Acupuncture needle stimulation on some physiological parameters after road transport and physical exercise in horse. Journal of Equine Veterinary Science, 48, 23-30. http://dx.doi.org/10.1016/j.jevs.2016.08.013. 
Rizzo, M., Arfuso, F., Giannetto, C., Giudice, E., Longo, F., Di Pietro, S., \& Piccione, G. (2017b). Cortisol levels and leukocyte population values in transported and exercised horses after acupuncture needle stimulation. Journal of Veterinary Behavior: Clinical Applications and Research, 18, 56-61. http://dx.doi.org/10.1016/i.jveb.2016.12.006.

Schoen, A. M. Veterinary acupuncture: ancient art to modern medicine. St. Louis: Mosby; 2001.

Soligo, M., Nori, S. L. L., Protto, V., Florenzano, F., \& Manni, L. (2013). Acupuncture and neurotrophinmodulation. In B. Y.Zeng, K. Zhao \& F. R. Liang (Eds), Neurobiology of acupuncture: 111 international review of neurobiology. London: Academic Press. http://dx.doi.org/10.1016/B978-0-12-411545-3.00005-5.

Suinn, R. M. M. (2005). Behavioral intervention for stress management in sports. International Journal of Stress Management, 12(4), 343-362. http://dx.doi.org/10.1037/1072-5245.12.4.343.

Teixeira, R. R., Diaz, M. M., Santos, T. V., Bernardes, J. T., Peixoto, L. G., Bocanegra, O. L., Bernardino Neto, M., \& Espindola, F. S. (2015). Chronic stress induces a hyporeactivity of the autonomic nervous system in response to acute mental stressor and impairs cognitive performance in business executives. PLoS One,10(3), e0119025. http://dx.doi.org/10.1371/journal.pone.0119025. PMid:25807003.

Villas-Boas, J. D., Dias, D. P. M., Trigo, P. I., Almeida, N. A. S., Almeida, F. Q., \& Medeiros, M. A. (2015). Acupuncture affects autonomic and endocrine but not behavioural responses induced by startle in horses. EvidenceBased Complementary and Alternative Medicine,2015, 9. http://dx.doi.org/10.1155/2015/219579. PMid:26413116.

Villas-Boas, J. D., Dias, D. P. M., Trigo, P. I., Almeida, N. A. S., Almeida, F. Q., \& Medeiros, M. A. (2016). Behavioural, endocrine and cardiac autonomic responses to a model of startle in horses. Applied Animal Behaviour Science, 174, 76-82. http://dx.doi.org/10.1016/j.applanim.2015.10.005.

Xie, H., \& Preast, V. Xie's veterinary acupuncture. Oxford: Blackwell; 2007. http://dx.doi.org/10.1002/9780470344569.

Yang, C. H., Lee, B., Jung, H., Shim, I., Roh, P., \& Golden, G. (2002). Effect of electroacupuncture on response to immobilization stress. Pharmacology, Biochemistry, and Behavior, 72(4), 847-855. http://dx.doi.org/10.1016/ s0091-3057(02)00769-4. PMid:12062574. 